\title{
The Heavens in August, 1921
}

\section{Dark Voids in the Starry Skies, and What They Mean}

By Prof. Henry Norris Russell, Ph.D.

$A^{\text {s we look out into the summer skies, on any clear }}$ the great brilliancy of the southern Milky Way. Almost from Altair to the horizon, there is a long succession of clouds and patches of light, whose brighter portions far exceed any other regions of the Galaxy which can be seen in our latitude.

Our first impression will probably be of the brightness, and the patchy character, of the luminosity. Upon a second and more careful survey, we will notice that all through this quarter of the heavens the Milky Way is double. Beside the conspicuous stream which first catches our eye there is another; further to our right, fainter, and in many places wider. Looking upward, we find that these two branches of the Milky Way may be traced northward beyond the celestial equator, and all the way into Cygnus, where they blend with the single stream which we know in the winter skies. In the opposite direction the division of the Galaxy extends down to our horizon, and far beyond. Southern observers can follow it up to the Cross, where again the single band of light replaces the double.

Between the two branches of the Milky Way the sky is dark-fully as dark as the background of hold in direct vision, one may notice sev-

higher up. a bar of cloud, is usually more conspicufound at mountain observatories, that the

\section{What the Camera Tells Us} hide it and in places practically blot it the heavens outside the Galaxy, and in places even darker. On a really clear night, when the zenith sky seems powdered with tiny stars, almost too faint for the eye to tiny stars, almost too faint for the eye to
hold in direct vision, one may notice several of these dark regions. The most conSagittarius, where, on the edge of the bright star-clouds, there is a dark region several degrees long from which lanes almost as dark run westward-one nearly toward Antares, the other a few degrees toward Antar extends almost across the Milky Way, like ous to one star-gazing, since it gets so much higher in the sky. It is only in clear, brilliant skies, such as may be found at mountain observatories, that the
unaided eye can do much to detect these unaided eye can do much to detect these
regions. A city sky, veiled with haze and illuminated by street lights, is almost hopeless.

But the real extent and nature of these dull patches in the heavens is revealed only by photography. For our present knowledge we have to thank primarily the skill'and assiduity of Professor Barnard, who has studied these objects for years, and gradually convinced the scientific world of the truth of his views.

Put briefly, his contention is this: that these dark regions are not holes in the star-clouds of the Milky Way, through which we see into the dark depths of space, but actual clouds of obscuring matspace, but actual clouds of obscuring matter, between us and the Milky Way, which
hide it and in places practically blot it

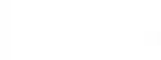

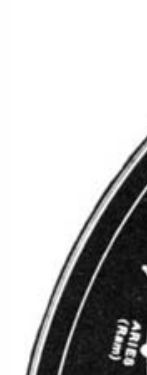

The reality of this obscuration first becomes apner between Sagittarius and Scorpio, in the southern part of Ophiuchus. Here the stars seems practically blotted out, so that there are regions where hardly one can be seen in the field of view of a great telescope-though, only a few degrees away, hundreds appear in a region of the sky of the same size.

It is only here and there that the "dark nebulae" are entirely opaque. More often a few scattering stars can be seen through them-probably greatly dimmed. They cannot be studied profitably with large telescopes usually sharp bit of detail. For the most part they are so big that only the wide-angle camera can display them, and a small instrument will then suffice. Photographs, exhibiting them as beautifully as the writer has ever known, have been shown by a colleague here, which were obtained with a hand camera (the lens of the highest grade), strapped to a telescope which served to keep it accurately pointed at the stars during exposures which often ran into many hours or even a ent in the case of the darkest markings, such as those - unless one wishes to examine some small and un- whole night. With such an equipment it is hard to find any large region of the Milky Way in which there is not some evidence of obscuration; and in many places
it is remarkable.

On looking at such a plate, one is moved to ask two questions. What are these vast dark clouds which come between us and the Milky Way? And where are they?

\section{Bright Nebulae and Dark Regions}

The second question is in some ways easier to answer. These obscured regions are permanent features of the heavens; hence they must be produced by something out among the stars. In many cases they are connected with regions of diffused visible nebulosity. For example, the dark lanes in Ophiuchus run up to the large patches of faint nebulosity which surround the stars Rho Ophiuchi and Nu Scorpii, in such a fashion that there can be no doubt that the luminous nebula is simply a part of the dark region, which is caused to shine for some reason connected with the presence of the stars about which the luminosity is condensed.

In these cases we may be practically sure that the however, probable that there are many other and more remote dark clouds, which look smaller because of their distance.

\section{The Nature of the Dark Clouds}

With regard to the true nature of these clouds, it is evident that they must be vast aggregates of some form of actual matter-and a very little consideration shows that the form of matter which is most effective must be fine dust. A cloud of fine particles-whether of actual dust or of the minute drops of water which constitute a fog-may be much more opaque in a few inches' thickness than all the many miles of air in our atmosphere. Larger particles, such as pebbles or rain drops, could also form an opaque cloud if there were enough of them; but calculation shows and common sense confirms that the same quantity of material, if broken up into smaller dust grains or drops, would suffice to make a far larger and denser cloud. If then in these dark nebulae we have actually a mixture of things, of all of gas-the fine dust particles and fine drops (of abou the size of a wave length of light) will be so much the best cloud-formers, pound for pound, that unless they are present in an extraordinarily small proportion they will account for most of the observed obscuration.

These clouds of cosmic dust, mixed with we know not how much gas or how many larger lumps of matter, are the hugest objects known to science. The great masses in Ophiuchus must be more than fifty light-years long and several lightyears wide. Those in Orion are probably bigger ; and Pannekoek estimates that the full extent of the cloud in Taurus is 200 light-years.

It is beginning to look probable that much, if not all, of the dark lane which runs down the Milky Way, and splits it in two for almost one-third of the circuit of the heavens, may be explained by similar obscuring matter, lying here and there, cloud behind cloud, and hiding from our eves perhaps the grandest part of the stellar universe. Such a cloud mass must be thousands of light-years in extent.

of course, these vast clouds are not solid. They are probably incomparably less substantial than the thinnest terrestrial fog-for a few rods of the latter will absorb more light than a billion miles of the former. How they got there we need hardly ask. Of all things visible they seem nearest to the primeval chaos. Indeed, they might well be described in the scruptural phrase "without form and void . . . darkness upon the face of the deep."

\section{The Heavens}

The region of the sky of which we have spoken is nearly in the south earlier in sizes-from great lumps of rock to separate molecules the evening, but well in the southwest at nebulae, both bright and dark, are at substantially the same distance as these stars. In this way we can say with some assurance that the obscuring clouds in Ophiuchus are at a distance of about 400 light-years. Another prominent group of regions of obscuration ap pears to be connected with the bright stars in Orion, and the great nebula there; and we may estimate it distance as 600 light-years

Still another such group lies in Taurus. The distance of this group, which is one of the largest and blackest of all, has been estimated by the Dutch astronomer Pannekoek in another way, by counting the number of faint stars per square degree in the dark regions and outside of them, and calculating, from our present knowledge of the distribution of the stars in space at what distance the absorbing screen must be in orde to obscure the observed proportions of the stars of the various magnitudes. He concludes that this screen is about 500 light-years distant.

These three great goscuring clouds are therefore nea us, in eomparison with the star-clouds of the Milky Way, whose distance may be roughly estimated as from twenty to fifty thousand light-years, if not more. It is, our hour of observation as indicated on the map. Scorpio is setting, with Sagittarius above on the left, and Aquila higher. Cygnus is right overhead; then, along the Galaxy, come Cepheus, Cassiopeia, and Perseus, low in the northeast. The Great Bear skims the northwestern horizon, with Draco and Ursa Minor above. Lyra is high in the west, with Hercules and Bootes below. Turning eastward we may see Pegasus and Andromeda, with Aquarius, Capricornus and the Southern Fish in the southeast.

\section{The Planets}

Mercury is a morning star at the beginning of the month, and rises at about 3.30 A. M. He soon draws in behind the sun and passes through conjunction on the 23rd, to reappear next month. Venus is a morning star in Gemini and Cancer, rising before $2 \mathrm{~A}$. M. at the beginning of the month, and about half an hour later at its close. Mars is a morning star, too, but too close to the sun to be easily visible.

Jupiter and' Saturn are evening stars and are getting closer together in the sky as they pass into the twilight. (Continued on page 108) 
Weber Crank-Pin Re-Turning Tool NO FILING NO OFFSE

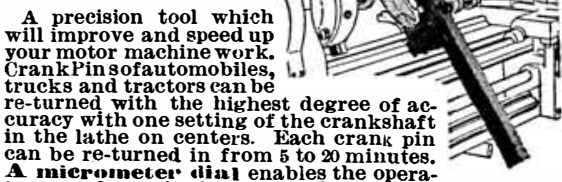

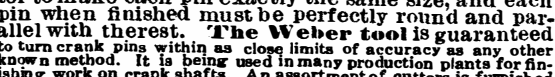
SAWYER - WEBER TOOL MFG. CO.
353 So. Alameda Street TOs Angeles, Calif.

For Gunsmiths, Tool Makers, Experimental \& Repair Work, etc.

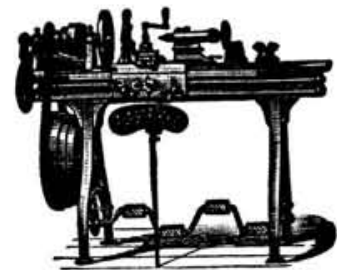

From 9-in. to 18-in. steam or Foot Power up Treadle.

W. F. \& J. Barnes $C_{0}$ 1999 Ruby Street

\section{ASBESTOS}

Wuantity. We produce all grades at our world famous BELL ASBESTOS MINES in Canada. We also carry

KEASBEY \& MATTISON COMPANY AMBLER, PENNA-1 U. S. A.

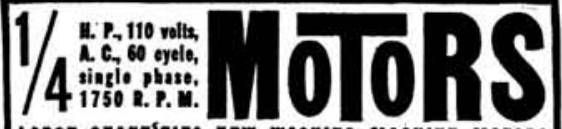

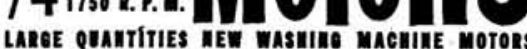

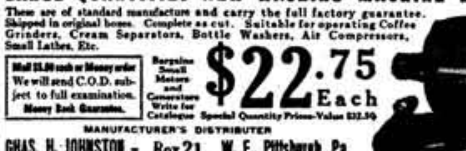

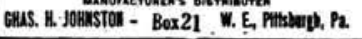

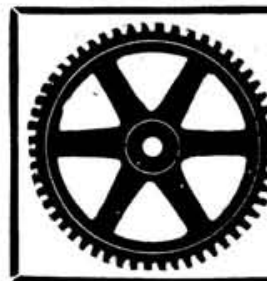

GEARS

All finds-small

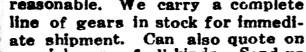

pour inquiritiof all kinds. Send a

Write for Catalogue

South Jeff arsoo Street Chicare

We Will Make It

Anything in a metal stamping or novelty pro-
duced from any metal and fi nished in any color.
Waterbury Button Co., Waterbury. Conn.

A New Book on a New Subject

BEHIND THE MOTION-

PICTURE SCREEN

By AUSTIN C. LESCARBOURA

$\mathrm{H}_{\text {screen. It takes the reader into that marvel- }}^{\mathrm{ERE} \text { at last, is the wonder book of the }}$ ous land where films are made, and where the

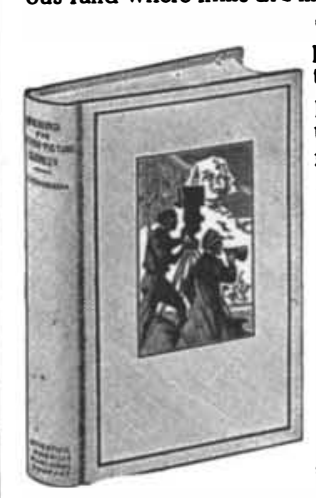
preme. Every step in photo-play is taken up in proper turn, and writing of the and writing of the jecting of the finished film on the screen in the picture. Talking pictures, natur-
al-color picclure,smicroolor picture, smicroture are treated upon in
due turn. This bock i $= \pm$

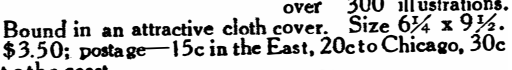
tothe coast.

One of the Most Attractive Books Ever Issued. $\underset{\substack{\text { Scientific American Publishing Co. } \\ \text { New York }}}{233 \text { Broadway }}$ large percentage of volatile matter, and during those periods when the boilers are being forced. An electric locomotive is used for the removal of ashes, running directly under the discharge gates.

The new plant is in the center of one of the busiest and most congested indusin its racts of Philadelphia, including known locally as Kensington and another district known as Richmond, given over largely to metal manufacturing plants. Power is supplied to these by underground cable at a potential of 13,200 volts and
stepped down at substations for other industrial and for lighting requirements.

In common with all the other buildings of the company this structure, when complete, will present a most pleasing and stately architectural appearance. John T. Windrim, the architect, has put into it the kind of thought and effort that was confined, a few years ago, to memorial buildings and the like. This is in line is based on the reasoning that the corporation is one of the city's biggest citizens and that it must do things in a big way. Few persons realize the tremendous amount of work and equipment involved in the distribution of electric energy. For this one new plant there are being installed more than sixty miles of underground high voltage cable, 219 miles of miles of overhead lines. Fach turbine in the plant is 48 feet long, 19 feet wide, 12 feet high and weighs 326 tons. and each is equipped with a 50,000 square foot condenser containing thirty-six miles of tubing, requiring 37,500 gallons of water per minute for condensing 369,000 pounds of steam every hour. For cooling the generators eight thousand cubic feet

\section{The Heavens in August, 1921}

Continued from page 100

At the beginning of the month they remain in sight until 9 P. M., but by its
close they are almost lost in the sun's close they are almost lost in the sun's
rays. Uranus is in Aquarius and comes to rays. Uranus is in Aquarius and comes to
opposition on the last day of the month. At that time he is in R. A. $22 \mathrm{~h} .38 \mathrm{~m}$. 35s., declination $9^{\circ} 27^{\prime}$ south, and is moving $9 \mathrm{~s}$. east and 53" south every day. He may be found $21 / 2^{\circ}$ west and $11 / 2^{\circ}$ south of the fourth magnitude star Lambda Aquarii, which is itself about one-third of the way from Beta Aquarii to Zeta Pegasi-these latter stars being marked on our map. Neptune is in conjunction
the 4 th, and is invisible.

The moon is new at 3 P. M. on the 3 rd, in her first quarter at $9 \mathrm{~A} . \mathrm{M}$. on the 10 th full at $10 \mathrm{~A}$. M. on the $18 \mathrm{th}$, and in her last quarter at $8 \mathrm{~A}$. M. on the 26th. She away on the 17th, and at her nearest again on the 31st. During the month she passes near Mercury and Mars on the 2nd, Neptune on the 3rd, Jupiter and Saturn on the 6th, Uranus on the 19th, Venus on the 30th, and Neptune and Mars on the 31.st.

Mount Wilson Observatory

July 1., 1921.

\section{A One-Piece Welded Structural} Frame

Continued from page 101)

more cheaply than either the screwed joint or the riveted or bolted joint. The matter of sanitation is simply one of trim construction that eliminates the ordinary recesses and

The adaptation of tubing to structural work was first conceived to meet the difficulty of obtaining building material in the oil fields during the recent adverse conditions that prevailed in the Southwest, and the first building erected was a comparatively small structure fabricated from odds and ends of pipe, of
which there is always an abundant supply in the oil districts. The success of this experimental step led to the erection of a larger building, the shop structure In this building, 50 by 100 feet, all of the joints in the tubing were welded. The side, ends and roof are of corrugated iron and the floor of concrete. The trusses were and tackle to the bottom chords, one at a
and time, and hoisting

ear of the ground.

The same company is now erecting still larger building in Dallas. This struc ture will be 100 by 200 feet. The sides and
ends will be of concrete, 10 inches in thickness, finished in marble; the roof will be sheathed with reinforced concrete slabs 2 inches thick; there will be a skylight 28 feet wide and two $2 \frac{1}{2}$-foot ventilator shafts, skylight and shafts extending the entire length of the building; the floor will be of concrete; and there will be steel ash windows and roller doors. A large chain hoist will be swung to one of the
trusses for the loading and unloading of trusses for the

This will be the largest building of its type yet erected, but it is stated that it y no means indicates the limit of dimen列 construction may be adrantageously
adapted; that any number of stories and adapted; that any number of stories and any size of floor space can be built in this
way. The new departure will be ohserved with considerable interest in building

\section{Sodium Hypochlorite}

THERE are signs that a new household bleaching, deodorizing and disinfecting agent is about to become popular in
the United States. It is called sodium the United States. It is called sodium
hypochlorite and is a close relative of chloride of lime which contains calcium hypochlorite.

Sodium and calcium hypochlorites are very interesting compounds, because they contain two active elements, oxygen and
chlorine, either of which without the other forms inert, stable compounds with other forms inert, stable compounds with
the sodium or calcium, but when both are present in the proportions to form hypochlorites they act as two husbands of a single wife would, if they were well matched in strength and little afraid of this family arrangement and look for a chance to escape and enter into more attractive combinations. Usually it is the oxygen that escapes, but when acids are
mixed with the hypochlorites, the chlomixed with the $h$
rine is driven out.

The oxygen in hypochlorites is loosely bound and very active. In the air, oxygen is rather sluggish unless the temperature is raised to the point where ordinary combustion takes place. We use matches to reach this temperature. But the oxygen of the air can be made active
at ordinary temperatures in another way. If sunlight and moisture are present, the energy of the sun tears apart the sluggish oxygen molecules and leaves the oxygen in an active form that will destroy germs, bleach colors and sweeten things that have foul smells. This is why we put linen
on the grass in the sunlight and sprinkle on the grass in the sunlight and sprinkle
it with water. The hypochlorites obtain their energy not from sunlight but from electricity. The firmly bound and sluggish chlorine in common salt is torn away and set free by electricity, and made to pass into a compound containing oxygen, such as soda
hypochlorites.

\section{Chloride of} very powerful antiseptic because it is used to sprinkle decaying vegetable and animal matter, it is put into ponds to disinfect them, which it does in such extreme dilutions as not to make the water
unfit to drink, and when it is mixed with a weak acid solution such as urine or a weak acid solution such as urine or
vinegar it generates chlorine enough to sweeten the air of a room without being
strong enough to irritate the lungs and throat. But for bleaching the wash, to
the take the place of sunlight, air and mois-

ure, chloride of lime is not well suited cause it hardens water and prevent the detergent action of soap. For this purpose the sodium hypochlorite is bet ter as it does not prevent the use of soap. It has been used for many years in the wash and for whitening and sweetenin wood floors and wood sinks.

Sodium hypochlorite will not remove all stains. There are some colors that are too fast to be destroyed by it, and this is really an advantage as it permits its use for bleaching the white part of printed calicoes and other partly colored cotton goods. The claims on the bottles come makes of hypochlorite are in exA canras tent left out in the air, moisture and sunlight for some years be comes rotten from the action of the oxygen of the air in excess. So too, if sodium hypochlorite is allowed to act too long on wash, or in too great concentrafabric, but if it is used with discretion according to the directions on the bottle, both as to strength and time, it will do more conveniently what air, sunshine and moisture do: bleach, sweeten and sterilize cotton and linen without destroying the fiber.

Sodium hypochlorite was used in the war to keep wounds aseptic, and it may hold aseptic. It is used in laundries, and is now being introduced for family use under many names, being easily identified by the peculiar and not disagreeable smell, which one soon learns to associate with cleanliness and purity. It is not a poisonous antiseptic like carbolic acid, corrosive sublimate and formaldehyde, un-
less it is mixed with large amounts of strong mineral acids, when chlorine is set free in such amounts as to be dangerous to breathe. Next to soap and washing soda, sodium hypochlorite may quite possibly become the most commonly Comsing reagent. from Value of Timber C

\section{P REJUDICE exists in certain quarters} dead trees, and some purchase specificaions insist that only timber cut from live trees will be acceptable. As a matter of fact when sound dead trees are sawed into lumber, and the weathered or charred outside is cut away, there is method known to the Forest Products distinguished from that cut from live trees, except that the lumber from dead 